\title{
S? \\ Charge fluctuations in nonlinear heat transport
}

\author{
Niklas M. Gergs, ${ }^{1}$ Christoph B. M. Hörig, ${ }^{1}$ Maarten R. Wegewijs, ${ }^{2,3,4}$ and Dirk Schuricht ${ }^{1}$ \\ ${ }^{1}$ Institute for Theoretical Physics, Center for Extreme Matter and Emergent Phenomena, Utrecht University, Leuvenlaan 4, \\ 3584 CE Utrecht, The Netherlands \\ ${ }^{2}$ Peter Grünberg Institut, Forschungszentrum Jülich, 52425 Jülich, Germany \\ ${ }^{3}$ Institute for Theory of Statistical Physics, RWTH Aachen University, 52056 Aachen, Germany \\ ${ }^{4}$ JARA-Fundamentals of Future Information Technology, Germany \\ (Received 25 July 2014; revised manuscript received 27 March 2015; published 26 May 2015)
}

\begin{abstract}
We show that charge fluctuation processes are crucial for the nonlinear heat conductance through an interacting nanostructure, even far from a resonance. We illustrate this for an Anderson quantum dot accounting for the first two leading orders of the tunneling in a master equation. The often made assumption that off-resonant transport proceeds entirely by virtual occupation of charge states, underlying exchange-scattering models, can fail dramatically for heat transport. The identified energy-transport resonances in the Coulomb blockade regime provide qualitative information about relaxation processes, for instance, by a strong negative differential heat conductance relative to the heat current. These can go unnoticed in the charge current, making nonlinear heat-transport spectroscopy with energy-level control a promising experimental tool.
\end{abstract}

DOI: 10.1103/PhysRevB.91.201107

PACS number(s): 73.23.Hk, 73.50.Lw, 73.63.-b

Recently, the experimental investigation of heat transport on the nanoscale has become possible [1-3]. These measurements are accurate enough to investigate the heat dissipation in molecular junctions with conductances as low as [4] $10^{-3} e^{2} / h$. Additionally, integrating energy-level control into thermoelectric junctions, e.g., by mechanical [5] gating, does not seem out of reach and, just recently, electrically gated thermoelectric nanojunctions have been demonstrated [6]. Here, by analyzing the generic effects of Coulomb interactions on the nonlinear heat transport in nanoscale systems, we will show that this is very promising.

Interaction effects have long been probed using gate controlled charge-current spectroscopy, a well-developed experimental tool to access the discrete quantum levels of nanostructures. Two prominent features in the charge current driven by a source-drain voltage underpin this successful method. The first is resonant or single-electron tunneling (SET), which depends on the level position relative to the electrochemical potential, $\mu_{\mathrm{R}}$ in Fig. 1(a): An electron jumps into or out of an orbital level, directly leading to a real change of its occupancy. The current shows sharp steps as new resonant transport processes are switched on with increasing bias. These processes are routinely identified in a three-terminal setup by plotting the charge conductance as function of the applied bias $V$ and the gate voltage, as exemplified in Fig. 2(a). Two-terminal measurements, e.g., using a scanning probe, correspond to line traces through such a plot. The second type of resonance is independent of the level position and appears as a horizontal line at $V=\Delta$ since it originates in the inelastic excitation by an energy $\Delta$ at fixed local electron number on the nanostructure. This off-resonant feature requires a second-order tunneling process in which an electron "scatters through," other charge states being only visited virtually [see Fig. 1(b)]. This is known as inelastic electron tunneling (IETS) [7,8] or inelastic cotunneling (ICOT) [9-12].

This inelastic tunneling resonance develops into a nonequilibrium Kondo resonance for low $\Delta$ and low temperatures [13$15]$, which is much sharper $[11,12]$ than the resonant tunneling feature corresponding to $\Delta$, providing better access to a range of physical phenomena in situ: an electronic level splitting (e.g., in a semiconductor nanostructure [16], carbon nanotube $[17,18]$, or a dopand atom [19]), a quantized vibrational frequency [20], or a spin splitting due to a magnetic field [8], exchange interaction [11,12,21], magnetic anisotropy (e.g., in molecules [22,23] or adatoms [24]), or spin-orbit coupling [25].

Thermoelectric transport has also been investigated within the two above-mentioned physical transport pictures. Theory mostly focused on the thermopower in the linear-response regime. This includes the study of resonant tunneling [26], inelastic tunneling [27-29], and Kondo processes [30-33]. Works addressing the nonlinear regime have either applied effective single-particle descriptions [34-38] or focused on thermoelectric devices close to resonance assuming weak tunneling [39-41] or a weak Coulomb interaction [42]. The heat current has received much less attention [3942]. A classification of nonlinear heat-transport features for a strongly interacting nanostructure going beyond weak tunneling, matching that of charge transport [43-45], still seems to be missing. This is important both for scanning probe setups [1-5] as well as thermoelectric setups [6,32] with energy-level control. In this Rapid Communication we address this problem and show that the heat current driven by a nonlinear electric and/or thermal bias contains qualitative information and deviates in a striking way from the charge transport, both in sign and amplitude. Its dependence on the level position reveals that relaxation processes of first order [Fig. 1(a)] in the tunneling can be very important for heat transport far from resonance (i.e., energy detuning larger than temperature). The crucial competition with an inelastic second-order excitation at finite voltage bias $V=\Delta$ [Fig. 1(b)] leads to real occupation of more than one charge state and is missed by inelastic transport theories relying on effective exchange- and potential-scattering amplitudes.

Model and method. To illustrate the generic picture of nonlinear thermoelectric transport through an interacting 
(a)
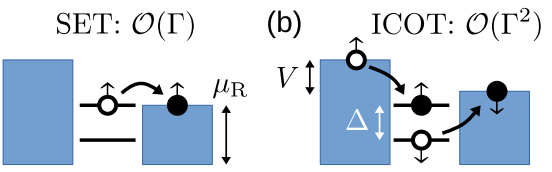

initial configuration

- final configuration

tunneling process

FIG. 1. (Color online) Examples of tunneling processes between electrodes (blue) and discrete quantum levels (black) (a) of first and (b) of second order in the tunneling rate $\Gamma$.

nanoscale object we analyze a resonant level with a strong Coulomb interaction and a well-defined spin-flip excitation $\Delta$ due to an external field. It is described by the Anderson quantum dot model $H_{\text {tot }}=H_{\mathrm{d}}+H_{\text {res }}+H_{\text {tun }}$ which also suffices to classify nonlinear thermoelectric transport features for more complex models [46]. These features should generally be observable in a range of nanostructures, at least for large level spacings and quasiregular electron filling of energy shells. The dot is described by $H_{\mathrm{d}}=\sum_{\sigma}(\varepsilon+\sigma \Delta / 2) d_{\sigma}^{\dagger} d_{\sigma}+$ $U N(N-1) / 2$, where $d_{\sigma}$ with $\sigma=\uparrow, \downarrow$ are the electron operators on the dot. Here $\varepsilon=\left(\varepsilon_{\uparrow}+\varepsilon_{\downarrow}\right) / 2$ is the orbital energy level and $\Delta=\varepsilon_{\uparrow}-\varepsilon_{\downarrow}$ denotes the energy of a local spin excitation for fixed $N=1$ due to a magnetic field, where $N=\sum_{\sigma} d_{\sigma}^{\dagger} d_{\sigma}$ is the electron number. Furthermore, $U$ is the strong Coulomb energy penalty paid when counting $N=2$ electrons in the shell. The electrodes, indexed by $\alpha=\mathrm{L}, \mathrm{R}$,

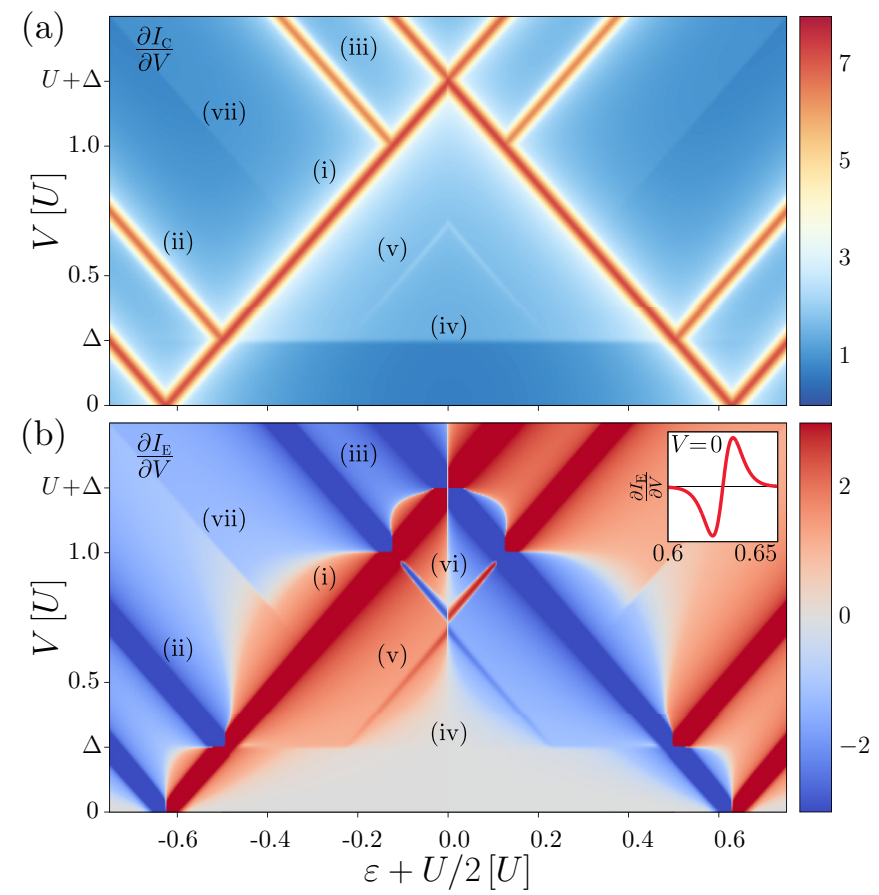

FIG. 2. (Color online) Transport through a quantum dot with interaction $U=\frac{1}{3} 10^{3} T$, inelastic excitation $\Delta=\frac{1}{4} U \approx$ 83.3T, and tunnel coupling $\Gamma=\frac{1}{3} 10^{-2} T$. (a) Charge conductance $\log _{10}\left(\left[\partial I_{\mathrm{C}} / \partial V\right] /\left[\Gamma^{2} / U^{2}\right]\right)$ and (b) energy conductance $\operatorname{slog}_{10}\left(\left[\partial\left(I_{\mathrm{E}} / \partial V\right)\right] /\left[\Gamma^{2} / U\right]\right)$ using the signed $\log , \operatorname{slog}_{10}(x):=$ $\operatorname{sgn}(x) \log _{10}(a|x|)$ for $a|x| \geqslant 10$ with $a=20$, linearized to $\operatorname{sog}_{10}(x):=a x / 10$ for $a|x| \leqslant 10$. Labels (i)-(vii) indicate the features discussed in the text but are not labeled at horizontally mirrored positions. Inset to (b): Linear energy conductance (a.u.) vs $\varepsilon+U / 2$ around the right SET resonance. are described as noninteracting reservoirs, $H_{\text {res }}=\sum_{\alpha} H_{\text {res }}^{\alpha}=$ $\sum_{\alpha k \sigma} \epsilon_{k} c_{\alpha k \sigma}^{\dagger} c_{\alpha k \sigma}$, with electron operators $c_{\alpha k \sigma}$. We allow for a nonlinear voltage bias $V$ between the reservoirs through their electrochemical potentials $\mu_{\mathrm{L}, \mathrm{R}}= \pm V / 2$ with temperatures $T_{\mathrm{L}}=T_{\mathrm{R}}=T$. (We comment on nonlinear thermal bias effects [46] later on.) The tunnel coupling has the generic form $H_{\text {tun }}=t \sum_{k \alpha \sigma}\left(c_{k \alpha \sigma}^{\dagger} d_{\sigma}+\right.$ H.c. $)$, the bare resonance width is given by the tunnel rate $\Gamma=2 \pi v_{0} t^{2}$, with $v_{0}$ the density of states in the reservoirs, and we set $e=\hbar=k_{\mathrm{B}}=g \mu_{\mathrm{B}}=1$.

We will only consider the stationary currents entering the right reservoir. The charge current is $I_{\mathrm{C}}=\left\langle\frac{d}{d t} N_{\text {res }}^{\mathrm{R}}\right\rangle$, where $N_{\text {res }}^{\mathrm{R}}$ is the electron number operator of the reservoir $\alpha=\mathrm{R}$. Similarly, the energy current is defined via $I_{\mathrm{E}}=\left\langle\frac{d}{d t} H_{\mathrm{res}}^{\mathrm{R}}\right\rangle$. The measurable heat current can be obtained via [47] $I_{\mathrm{Q}}=$ $I_{\mathrm{E}}-\mu_{\mathrm{R}} I_{\mathrm{C}}$. Since experimentally the way the voltage is applied is known (here $\mu_{\mathrm{R}}=-V / 2=-\mu_{\mathrm{L}}$ ) and the conserved charge current is available, the conversion from $I_{\mathrm{Q}}$ to $I_{\mathrm{E}}$ amounts to a simple background subtraction. Below we focus on the contribution $I_{\mathrm{E}}$ since it contains all interesting physical features. Also, $I_{\mathrm{C}}$ and $I_{\mathrm{E}}$ are more easily compared, highlighting the differences between charge and heat transport most directly, in particular, the bias and gate voltage dependence on which we focus here. We use $U$ as the unit of energy; in experiments it is readily obtained from the height of the Coulomb diamond (cf. Fig. 2) and ranges from 0.1 to $10 \mathrm{meV}$ in the semiconductor [16] and carbon nanotube quantum dots $[17,18]$ to $10-100 \mathrm{meV}$ in the molecular [20] and atomic quantum dots [19,24]. The effects in the currents that we focus on below scale as $\partial I_{\mathrm{E}} / \partial V \propto U \partial I_{\mathrm{C}} / \partial V=\Gamma^{2} / U$ for the parameter regime of interest $\Gamma \ll U$ when fixing $\Delta$ relative to $U$. Estimations based on this [46] indicate that the predicted energy currents may be in the range of experimental resolution of tens of $\mathrm{nW}$, in particular, in molecular junctions.

The currents and the underlying nonequilibrium dot-state occupations are calculated using a reduced density-operator transport theory $[48,49]$ accounting for the strong local interaction $U$. This approach is perturbative in the tunneling rates and well controlled in the regime $\Gamma \ll T$. While keeping this restriction we recover [46] for $U=0$ the corresponding results of the Landauer approach $[4,38]$. We go beyond standard approaches by including the competition of all tunneling rates $\mathcal{O}(\Gamma)$ and $\mathcal{O}\left(\Gamma^{2}\right)$ (Fig. 1) into the stationary master equation $\dot{p}=0=W p$ for the occupations $p=\left(p_{0}, p_{\uparrow}, p_{\downarrow}, p_{\uparrow \downarrow}\right)$ (see Ref. [46] for more details of the calculations [45,50,51] of the transition rate matrix $W$ and the current). We focus on the dominant energy dependence introduced by the interacting quantum dot, assuming a flat spectral density in the wide-band limit for the electrodes.

$\mathcal{O}(\Gamma)$ effects. A first glance at the charge and energy conductance plotted in Figs. 2(a) and 2(b), respectively, already reveals that the energy-transport spectrum is much richer: There is a significant gain in the contrast due to its many sign changes. In these plots the stage is set by resonant tunneling features due to the processes of $\mathcal{O}(\Gamma)$ [Fig. 1(a)] which are well understood [46]. These occur when one of the four single-electron addition energies $\varepsilon_{\sigma}$, and $\varepsilon_{\sigma}+U(\sigma=\uparrow$ , $\downarrow)$ matches $\mu_{\mathrm{L}, \mathrm{R}}= \pm V / 2$. This happens, e.g., at the lines labeled (i)-(iii) in Fig. 2. Indicated by (i) are resonant tunneling transitions between the ground states of subsequent electron 

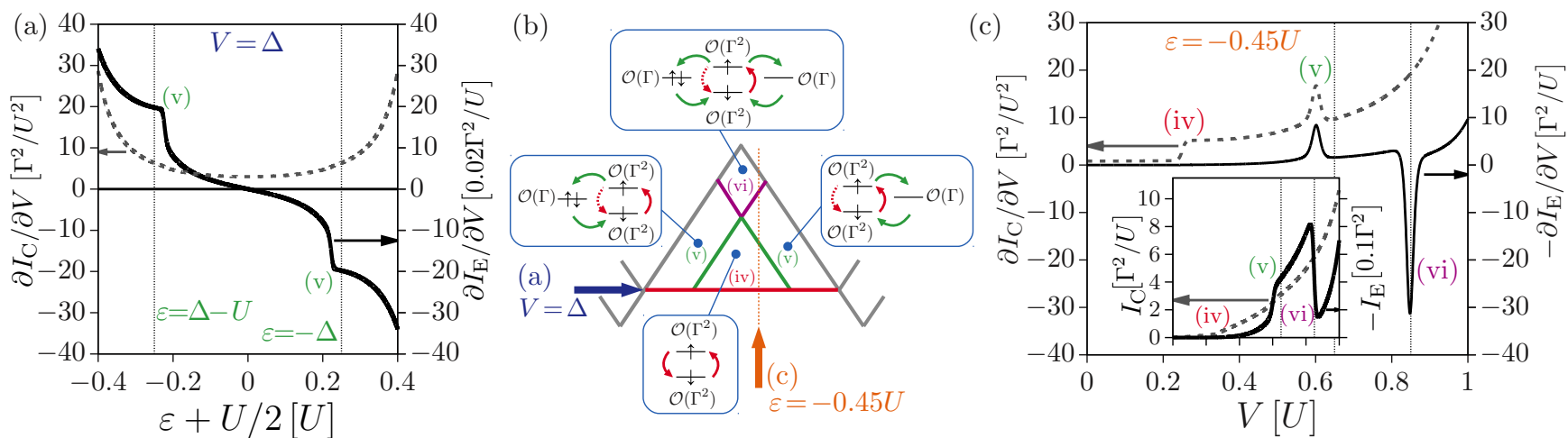

FIG. 3. (Color online) Explanation of Fig. 2. In (b) we sketch the stability diagrams of Fig. 2 showing by blue and orange arrows where the cuts in (a) and (c) are taken. (a) and (c) show $\partial I_{\mathrm{C}} / \partial V$ (dashed) and $\partial I_{\mathrm{E}} / \partial V$ (solid) as functions of the level position $\varepsilon$ for fixed $V$ and vice versa, respectively, and the vertical dotted lines indicate COSET resonance positions. In (c) we plot the negative of $\partial I_{\mathrm{E}} / \partial V$ and $I_{\mathrm{E}}$ for clarity. In (b), the boundaries of various regimes (iv)-(vi) and the corresponding processes discussed are shown in the inset boxes. The gray lines are the well-known SET resonances. The horizontal red line is the $V=\Delta$ threshold for ICOT excitation [Fig. 1(b)] shown in the inset box to (iv). When crossing from (iv) either of the green lines, a single two-step relaxation path is switched on (COSET), colored green in the inset to regimes (v). When subsequently crossing the purple lines (vi) both these green relaxation paths become active, as shown in the inset box to (vi).

numbers $N$ of the dot $(0 \rightarrow 1$ and $1 \rightarrow 2)$. As shown in the inset in Fig. 2(b), there are sawtooth-shaped resonances [26] in $\partial I_{\mathrm{E}} / \partial V$ as functions of the level position $\varepsilon$ corresponding to the Coulomb peaks in $\partial I_{\mathrm{C}} / \partial V$ associated with one-electron processes $|\downarrow\rangle \rightarrow|0\rangle$ and $|\downarrow\rangle \rightarrow|\uparrow \downarrow\rangle$, respectively. The sign change in $\partial I_{\mathrm{E}} / \partial V$ reflects that excess energy is carried by electrons or holes. This basic energy-transport feature reappears at several positions in Fig. 2(b), e.g., also when at (ii) a resonant tunneling process additionally excites the dot or at (iii) such a process starts off in the $N=1$ excited state $|\uparrow\rangle$, the process of Fig. 1(a). The broadening of these resonant tunneling lines is determined by the temperature for $T \gg \Gamma$.

$\mathcal{O}\left(\Gamma^{2}\right)$ effects. Qualitative differences show up inside the central off-resonant regime-opened up by the Coulomb interaction $U$-where the simple resonant picture just discussed breaks down. Here coherent electron-hole processes of $\mathcal{O}\left(\Gamma^{2}\right)$ that leave $N$ fixed, such as Fig. 1(b), become important as well. These give rise to qualitatively different effects not captured by $\mathcal{O}(\Gamma)$ master equations or even approaches that also include tunnel broadening and shifts [37]. For voltages $V \lesssim \Delta=\varepsilon_{\uparrow}-\varepsilon_{\downarrow}$ only elastic $\mathcal{O}\left(\Gamma^{2}\right)$ tunneling processes are possible which produce a smooth nonexponential background in both $\partial I_{\mathrm{C}} / \partial V$ (qualitatively similar to that found for metallic islands $[27,29])$ and $\partial I_{\mathrm{E}} / \partial V$. However, above the threshold line $V=\Delta$, indicated in red by (iv) in the schematic Fig. 3(b), a different inelastic tunneling process $\mathcal{O}\left(\Gamma^{2}\right)$ sets in: Electrons tunnel onto and off the dot while depositing an energy $\Delta$, as sketched in Fig. 1(b). This yields the characteristic step in the charge conductance [8] in Fig. 2(a) at $V=\Delta$ all across the off-resonant regime [11]. Our calculations show that the energy conductance $\partial I_{\mathrm{E}} / \partial V$ also shows such an inelastic tunneling feature at the corresponding line (iv) in Fig. 2(b). As expected, it changes sign when the electron and hole processes change roles, similar to the sawtooth-shaped resonances discussed above, but now when tuning the level position through the center of the off-resonant regime $(\varepsilon=-U / 2)$. Inspection of the magnitude of the inelastic step at $V=\Delta$ as a function of the level position $\varepsilon$ in Fig. 3(a) reveals a dramatic difference: Whereas the charge conductance amplitude at $V=\Delta$ is smooth and featureless as $\varepsilon$ is varied, the energy conductance amplitude sharply drops at $(\mathrm{v})$ when $\varepsilon \approx-\Delta$ or $\varepsilon+U \approx \Delta$. This big difference also shows up in Fig. 2(b) where the central part of the horizontal inelastic tunneling onset is completely missing, in contrast to Fig. 2(a). The strong reduction of $\partial I_{\mathrm{E}} / \partial V$ when entering the central region is remarkable: Everywhere in Fig. 3(a) we are still far from resonance, i.e., $\left|\varepsilon-\mu_{\mathrm{L}}\right|,\left|\varepsilon+U-\mu_{\mathrm{R}}\right| \gg T \gg \Gamma$. There is a second regime where the behavior of the energy conductance radically deviates from that of the charge conductance: The bias dependence plotted in Fig. 3(c) shows at (vi) a strong negative differential energy conductance $\partial I_{\mathrm{E}} / \partial V$ relative to the energy current $I_{\mathrm{E}}$ by far exceeding the feature (v) discussed below in magnitude. As the inset indicates, after encountering the large energy current change at $(\mathrm{v})$, the energy current drops back at (vi). Also this shows up in Fig. 2(b) as sharp blue (red) boundaries of the diamond-shaped region containing the label (vi) on the red (blue) background. The charge conductance in Fig. 3(c) is again featureless there. We now explain in three steps (A)-(C) how these dramatic differences come about physically, by following the vertical line in the schematic Fig. 3(b) at $\varepsilon=-0.45 U$. Our discussion explains which processes lead to changes in the occupations and transport rates [46] and are substantiated by numerical calculations.

(A) Starting in the state $|\downarrow\rangle$ at $V=0$ and increasing the bias, we first hit the red threshold (iv) at $V=\Delta$. Beyond this line the excited state $|\uparrow\rangle$ becomes occupied by inelastic tunneling [Fig. 1(b)] and it relaxes by similar inelastic processes. These transitions are indicated by the red arrows in the lower inset in Fig. 3(b). This gives an increased charge conductance as the electrons find an additional path through the quantum dot while keeping the charge fixed to $N=1$, only virtually visiting other charge states $N=0,2$. In contrast, the energy current is relatively low due to two effects: First, real charge fluctuations are suppressed $(N=1)$ and only inelastic tunneling processes $|\downarrow\rangle \leftrightarrow|\uparrow\rangle$ occur, the rates for both of which are $\propto \Gamma^{2}$, much smaller than the rates 
$\mathcal{O}(\Gamma)$. Second, there is a significant partial cancellation of the energy currents of these $\mathcal{O}\left(\Gamma^{2}\right)$ processes, namely, of a positive contribution due the inelastic tunneling relaxation process $|\uparrow\rangle \rightarrow|\downarrow\rangle$, and a negative contribution due to the inelastic tunneling excitation process $|\downarrow\rangle \rightarrow|\uparrow\rangle$ [Fig. 1(b)]. This relates to the generic electron-hole symmetry between two Coulomb-split SET resonances associated with filling a single orbital shell, captured by our model [46].

(B) Increasing $V$ further and crossing the green line (v) in Fig. 3(b), a two-step resonant tunneling relaxation path is switched on, indicated by the green arrows in the right inset. This sharply increases the magnitude of the energy current at (v) in Fig. 3(c) since it lifts the above cancellation of opposing energy currents of comparable magnitude: The inelastic tunneling relaxation process (red dashed downward arrow) is overridden by a much faster $\mathcal{O}(\Gamma)$ two-step resonant tunneling relaxation (green arrows), $|\uparrow\rangle \rightarrow|0\rangle$ of Fig. 1(a) followed by $|0\rangle \rightarrow|\downarrow\rangle$. This composite mechanism is called cotunnelingassisted SET [43,45,51,52] (COSET) and involves the real occupation of the $N=0$ state, despite the prevalence of $N=1$ states due to the Coulomb blockade.

(C) When $V$ finally crosses the blue line (vi) in Fig. 3(b), the other green relaxation path via the $N=2$ state also becomes active, as the upper inset shows. Although still far from resonance, both the $N=0$ and $N=2$ charge states become occupied for real because both $\mathcal{O}(\Gamma)$ relaxation pathways are turned on. Remarkably, this increased relaxation does not increase the energy current as above, but instead suppresses it. The reason is that the signed energy-current contributions from the two $\mathcal{O}(\Gamma)$ pathways now cancel each other-in contrast to case (B) where only one such pathway is active-thereby strongly reducing the energy current.

Discussion. We establish the complete classification of the nonlinear energy transport by noting the additional feature due to the tunneling of pairs [50] of either electrons or holes, which is again more prominent in the energy conductance [line (vii) in Fig. 2]. Much of the above remains qualitatively the same when including a junction and spin [53-56] dependence of the tunneling constants $\Gamma_{\alpha \sigma}$, or a combined voltage $\left(\mu_{\mathrm{L}}>\mu_{\mathrm{R}}\right)$ and thermal bias [39-41] $\left(\Gamma \ll T_{\mathrm{L}}<T_{\mathrm{R}} \ll U\right)$ : Interestingly, in the latter case the COSET resonances may be used experimentally to estimate the thermal gradient in situ [46].

The above described nonequilibrium competition between real and virtual processes, together with the sign of the energy currents, leads to an unexpectedly rich energy current spectrum. Importantly, for more complex multilevel quantum dots, the above identified elementary signatures are just repeated every time a different electronic orbital is filled when scanning the gate voltage. Our model captures this generic pattern which is well attested experimentally for the charge current. However, we even find [46] that several replicas of these features can appear in energy transport inside the Coulomb blockade regime (e.g., as negative $\partial I_{\mathrm{E}} / \partial V$ relative to $I_{\mathrm{E}}$ ), but also outside, at higher voltage, again in stark contrast to charge transport. Combining a three-terminal setup with measurements of the energy conductance may thus reveal new qualitative information about the relaxation processes. The much enhanced effect of COSET at (v) and (vi) in the energy transport of Fig. 2(b) should be experimentally accessible since even the weaker COSET features in the charge transport of Fig. 2(a) have been measured $[17,18,43]$.

Our results also indicate that the analysis of two-terminal thermoelectric measurements requires extra care due to the lack of gate-spectroscopic information. Often the two regimes of pure inelastic tunneling [label (iv) in Fig. 3(b)] and COSET [label (v)] are not distinguished. For the charge conductance this may not seem so important, but our results show that for the energy conductance this distinction is absolutely vital. Theoretical descriptions used to model scanning-probe experiments and quantum dots in the Coulomb blockade regime are often based on effective models including only effective exchange- and potential-scattering terms. These may fail badly for the energy current since they include only inelastic tunneling (keeping $N=1$ fixed) and eliminate the important real charge fluctuations (to $N=0$ and 2) involved in COSET. In Fig. 2(b) such an approximation is suitable only in a limited regime [the triangle labeled (iv)]. Extending the model to include more inelastic excitations further narrows it down [46]. Nonlinear energy transport thus requires careful consideration: One needs a physical model allowing for charge fluctuations as well as a nonequilibrium transport theory that captures at least the first two leading orders of tunneling processes for strong interactions. Experimentally, even higher-order tunneling effects may be important [37] and it is of interest to explore renormalization effects $[12,57,58]$.

We thank C. Cuevas, D. DiVincenzo, F. Haupt, M. Hell, M. Leijnse, P. Reddy, and R. Saptsov for valuable discussions. N.M.G., C.B.M.H., and D.S. thank the Institute for Theory of Statistical Physics, RWTH Aachen University, where substantial parts of this work have been performed. This work is part of the D-ITP consortium, an NWO program funded by the Dutch Ministry of Education, Culture and Science (OCW). C.B.M.H. and D.S. were supported by the DFG through the Emmy-Noether Program under SCHU 2333/2-1.
[1] P. Reddy, S.-Y. Jang, R. A. Segalman, and A. Mujamdar, Science 315, 1568 (2007).

[2] K. Baheti, J. A. Malen, P. Doak, P. Reddy, S.-Y. Jang, T. D. Tilley, A. Mujamdar, and R. A. Segalman, Nano Lett. 8, 715 (2008).

[3] J. R. Widawsky, P. Darancet, J. B. Neaton, and L. Venkataraman, Nano Lett. 12, 354 (2012).

[4] W. Lee, K. Kim, W. Jeong, L. A. Zotti, F. Pauly, J. C. Cuevas, and P. Reddy, Nature (London) 498, 209 (2013).
[5] R. Temirov, A. Lassise, F. B. Anders, and F. S. Tautz, Nanotechnology 19, 065401 (2008).

[6] Y. Kim, W. Jeong, K. Kim, W. Lee, and P. Reddy, Nat. Nanotechnol. 9, 881 (2014).

[7] J. Lambe and R. C. Jaklevic, Phys. Rev. 165, 821 (1968).

[8] A. J. Heinrich, J. A. Gupta, C. P. Lutz, and D. M. Eigler, Science 306, 466 (2004).

[9] L. I. Glazman and K. A. Matveev, JETP Lett. 51, 484 (1990) [Pis'ma Zh. Eksp. Teor. Fiz. 51, 425 (1990)]. 
[10] D. V. Averin and Y. V. Nazarov, Phys. Rev. Lett. 65, 2446 (1990).

[11] S. De Franceschi, S. Sasaki, J. M. Elzerman, W. G. van der Wiel, S. Tarucha, and L. P. Kouwenhoven, Phys. Rev. Lett. 86, 878 (2001).

[12] J. Paaske, A. Rosch, P. Wölfle, N. Mason, C. M. Marcus, and J. Nygård, Nat. Phys. 2, 460 (2006).

[13] A. Rosch, J. Paaske, J. Kroha, and P. Wölfle, Phys. Rev. Lett. 90, 076804 (2003).

[14] S. Kehrein, Phys. Rev. Lett. 95, 056602 (2005).

[15] H. Schoeller and F. Reininghaus, Phys. Rev. B 80, 045117 (2009); 80, 209901(E) (2009).

[16] D. M. Zumbühl, C. M. Marcus, M. P. Hanson, and A. C. Gossard, Phys. Rev. Lett. 93, 256801 (2004).

[17] S. Sapmaz, P. Jarillo-Herrero, J. Kong, C. Dekker, L. P. Kouwenhoven, and H. S. J. van der Zant, Phys. Rev. B 71, 153402 (2005).

[18] A. K. Hüttel, B. Witkamp, M. Leijnse, M. R. Wegewijs, and H. S. J. van der Zant, Phys. Rev. Lett. 102, 225501 (2009).

[19] F. A. Zwanenburg, A. S. Dzurak, A. Morello, M. Y. Simmons, L. C. L. Hollenberg, G. Klimeck, S. Rogge, S. N. Coppersmith, and M. A. Eriksson, Rev. Mod. Phys. 85, 961 (2013).

[20] E. A. Osorio, K. O’Neill, N. Stuhr-Hansen, O. F. Nielsen, T. Bjørnholm, and H. S. J. van der Zant, Adv. Mater. 19, 281 (2007).

[21] J. E. Grose, E. S. Tam, C. Timm, M. Scheloske, B. Ulgut, J. J. Parks, H. D. Abruña, W. Harneit, and D. C. Ralph, Nat. Mater. 7, 884 (2008).

[22] J. J. Parks, A. R. Champagne, G. R. Hutchison, S. Flores-Torres, H. D. Abruña, and D. C. Ralph, Phys. Rev. Lett. 99, 026601 (2007).

[23] A. S. Zyazin, J. W. G. van den Berg, E. A. Osorio, H. S. J. van der Zant, N. P. Konstantinidis, M. Leijnse, M. R. Wegewijs, F. May, W. Hofstetter, C. Danieli, and A. Cornia, Nano Lett. 10, 3307 (2010).

[24] S. Loth, K. von Bergmann, M. Ternes, A. F. Otte, C. Lutz, and A. Heinrich, Nat. Phys. 6, 340 (2010).

[25] T. Jespersen, K. Grove-Rasmussen, J. Paaske, K. Muraki, T. Fujisawa, J. Nygård, and K. Flensberg, Nat. Phys. 7, 348 (2011).

[26] C. W. J. Beenakker and A. A. M. Staring, Phys. Rev. B 46, 9667 (1992).

[27] M. Turek and K. A. Matveev, Phys. Rev. B 65, 115332 (2002).

[28] J. Koch, F. von Oppen, Y. Oreg, and E. Sela, Phys. Rev. B 70, 195107 (2004).

[29] B. Kubala and J. König, Phys. Rev. B 73, 195316 (2006).

[30] T. A. Costi and A. C. Hewson, J. Phys.: Condens. Matter 5, L361 (1993).

[31] T.-S. Kim and S. Hershfield, Phys. Rev. Lett. 88, 136601 (2002).

[32] R. Scheibner, H. Buhmann, D. Reuter, M. N. Kiselev, and L. W. Molenkamp, Phys. Rev. Lett. 95, 176602 (2005).

[33] T. A. Costi and V. Zlatić, Phys. Rev. B 81, 235127 (2010).

[34] M. Galperin, M. A. Ratner, and A. Nitzan, J. Phys.: Condens. Matter 19, 103201 (2007).

[35] R. López and D. Sánchez, Phys. Rev. B 88, 045129 (2013).

[36] R. S. Whitney, Phys. Rev. B 88, 064302 (2013).

[37] S. Fahlvik Svensson, E. A. Hoffmann, N. Nakpathomkun, P. M. Wu, H. Q. Xu, H. A. Nilsson, D. Sánchez, V. Kashcheyevs, and H. Linke, New J. Phys. 15, 105011 (2013).

[38] L. A. Zotti, M. Bürkle, F. Pauly, W. Lee, W. J. K. Kim, Y. Asai, P. Reddy, and J. C. Cuevas, New J. Phys. 16, 015004 (2014).
[39] M. Esposito, K. Lindenberg, and C. van den Broeck, Europhys. Lett. 85, 60010 (2009).

[40] M. Leijnse, M. R. Wegewijs, and K. Flensberg, Phys. Rev. B 82, 045412 (2010).

[41] H. Wang, G. Wu, Y. Fu, and D. Chen, J. Appl. Phys. 111, 094318 (2012).

[42] D. M. Kennes, D. Schuricht, and V. Meden, Europhys. Lett. 102, 57003 (2013)

[43] R. Schleser, T. Ihn, E. Ruh, K. Ensslin, M. Tews, D. Pfannkuche, D. C. Driscoll, and A. C. Gossard, Phys. Rev. Lett. 94, 206805 (2005).

[44] J. Koch, F. von Oppen, and A. V. Andreev, Phys. Rev. B 74, 205438 (2006).

[45] M. Leijnse and M. R. Wegewijs, Phys. Rev. B 78, 235424 (2008).

[46] See Supplemental Material at http://link.aps.org/supplemental/ 10.1103/PhysRevB.91.201107 for (1) details on the used theoretical method; and (2) additional information including a discussion of results obtained for multilevel systems. For specific aspects, see also Refs. [4,29,34-36,38-41,44,45,4859].

[47] F. Giazotto, T. T. Heikkilä, A. M. S. A. Luukanen, and J. P. Pekola, Rev. Mod. Phys. 78, 217 (2006).

[48] H. Schoeller, Eur. Phys. J.: Spec. Top. 168, 179 (2009).

[49] R. B. Saptsov and M. R. Wegewijs, Phys. Rev. B 86, 235432 (2012).

[50] M. Leijnse, M. R. Wegewijs, and M. H. Hettler, Phys. Rev. Lett. 103, 156803 (2009).

[51] S. Koller, M. Grifoni, M. Leijnse, and M. R. Wegewijs, Phys. Rev. B 82, 235307 (2010).

[52] V. N. Golovach and D. Loss, Phys. Rev. B 69, 245327 (2004).

[53] M. Pustilnik, Y. Avishai, and K. Kikoin, Phys. Rev. Lett. 84, 1756 (2000).

[54] J. König, J. Martinek, J. Barnaś, and G. Schön, in CFN Lectures on Functional Nanostructures, edited by K. Busch et al., Lecture Notes in Physics Vol. 658 (Springer, Berlin, 2005).

[55] J. Paaske, A. Andersen, and K. Flensberg, Phys. Rev. B 82, 081309(R) (2010).

[56] M. Pletyukhov and D. Schuricht, Phys. Rev. B 84, 041309(R) (2011).

[57] M. Pletyukhov and H. Schoeller, Phys. Rev. Lett. 108, 260601 (2012).

[58] A. V. Kretinin, H. Shtrikman, and D. Mahalu, Phys. Rev. B 85 , 201301(R) (2012)

[59] R. B. Saptsov and M. R. Wegewijs, Phys. Rev. B 90, 045407 (2014); H. Schoeller, Interactions and Transport Properties (Springer, Berlin, 1999), p. 137; Low-Dimensional Systems. Interactions and Transport Properties, edited by T. Brandes, Lecture Notes in Physics Vol. 544 (Springer, Berlin, 2000), p. 137; E. Bonet, M. M. Deshmukh, and D. C. Ralph, Phys. Rev. B 65, 045317 (2002); C. Romeike, M. R. Wegewijs, and H. Schoeller, Phys. Rev. Lett. 96, 196805 (2006); M. M. Deshmukh, E. Bonet, A. N. Pasupathy, and D. C. Ralph, Phys. Rev. B 65 , 073301 (2002); C. Stevanato, M. Leijnse, K. Flensberg, and J. Paaske, ibid. 86, 165427 (2012); M. Hell, B. Sothmann, M. Leijnse, M. R. Wegewijs, and J. König, ibid. 91, 195404 (2015); B. Muralidharan and M. Grifoni, ibid. 88, 045402 (2013); B. Wunsch, M. Braun, J. König, and D. Pfannkuche, ibid. 72, 205319 (2005); A. Donarini, B. Siegert, S. Sobczyk, and M. Grifoni, ibid. 86, 155451 (2012); D. Darau, G. Begemann, A. Donarini, and M. Grifoni, ibid. 79, 235404 (2009). 